\title{
STUDIES IN THEORETICAL HIGH ENERGY PARTICLE PHYSICS
}

\author{
Technical Progress Report
}

Henrik Aratyn, Lee Brekke, Wai-Yee Keung, Prasanta Panigrahi, and Uday Sukhatme Department of Physics

University of Illinois at Chicago

Chicago, Illinois 60680

\section{DISCLAIMER}

This report was prepared as an account of work sponsored by an agency of the United States Government. Neither the United States Government nor any agency thereof, nor any of their employees, makes any warranty, express or implied, or assumes any legal liability or responsibility for the accuracy, completeness, or usefulness of any information, apparatus, product, or process disclosed, or represents that its use would not infringe privately owned rights. Reference herein to any specific commercial pruduct, process, or service by trade name, trademark, manufacturer, or otherwise does not necessarily constitute or imply its endorsement, recommendation, or favoring by the United States Government or any agency thereof. The views and opinions of authors expressed herein do not necessarily state or reflect those of the United States Goverriment or any agency thereof.

\section{November 1990}

\section{Prepared for}

THE U.S. DEPARTMENT OF ENERGY

AGREEMENT NR. DE-FG02-84ER40173 


\section{ABSTRACT}

This is a technical progress report for grant no. DE-FG02-84ER40173 describing research carried out in 1989-1990.

NOTICE

This report was prepared as an acccunt of work sponsored by the United States Government. Neither the United Siates nor the Department of Energy, nor any of their employees, nor any of their contractors, subcontractors, or their employees, makes arıy warranty, express or implied, or assumes sny legal llability or responsibility for the accuracy, completeness, or usefulness of any information, apparatus, product or process disclosed or represents that its use would not infringe privately-owned rights. 


\section{TECHNICAL PROGRESS REPORT (Grant NO. DE-FG02-84ER40173)}

In this report, we describe technical progress achleved in various areas of research in 1989-1990. A detalled account of our work can be found in the following articles, which have either been published or circulated in preprint form. The publications are labeled by the names of physicists in our group $[A \equiv$ Aratyn, $B \equiv$ Brekke, $K \equiv$ Keung, $P \equiv$ Panigrahi, $S \equiv$ Sukhatme].

A1. Abelized Virasoro Operators, Geometry and the String Vertex Operator Aratyn, R. Ingermanson

Published in Int. J. Mod. Phys. A4, 3503-3575 (1989)

A2. Covariant BRS Quantization of the $100 \mathrm{~N}=1$ Brink-Schwarz Superparticle Aratyn and $R$. Ingermanson

Published in Phys. Rev. D39, 503-508 (1989)

A3. On the Geometry of the Graded Constraint Algebras; Vielbeins, Metric Tensors and the String Vertex Superoperators

Aratyn

Published in Mod. Phys. Lett. A4, 1667-1679 (1989)

A4. BRS Cohomology in Terms of the Abelian String Operators Aratyn

Published in Classical and Quantum Gravity 6,. L125-L131 (1989)

A5. BRS Cohomology in String Theory; Geometry of Abelization and the Quartet Mechanism

Aratyn

Published in Journal of Mathematical Physics 31, 1240-1252 (1990)

A6. A Geometric Approach to the String BRS Cohomology

Aratyn

Talk at the XVII ${ }^{\text {th }}$ International Conference on Differential Geometric Methods in Theoretical Physics: "Physics and Geometry", Tahoe Clty, CA, July 2-8, 1989, In Differential Geometric Methods in Theoretical Physics, Plenum, N.Y. (1990) 423-435

A7. Superspace Actions on Coadjoint Orbits of Graded Infinite-Dimensional Groups Aratyn, E. Nissimov, S. Pacheva and S. Solomon

Published in Phys. Lett. 234B, 307-314 (1990)

A8. Symplectic A.ctions on Coadjoint Orbits

Aratyn, E. Nissimov, S. Pacheva and A. H. Zimerman

Published in Phys. Lett. 240B, 127-132 (1990)

A9. Noether Theorem for Geometric Actluns and the Area Preserving Diffeomorphisms on Torus

Aratyn, E. Nissimov, S. Pacheva and A.H. Zimerman

Published in Phys. Lett. 242B, 377-382 (1990) 
A10. On the Group Theoretical Meaning of the Conformal Field Theories in the Framework of Coadjoint Orbits

Aratyn, E. Nissim and S. Pacheva

UICHEP-TH/90-6

May 1990, 6pp, to be published in Phys. Lett. B

A11. The Hidden Kac-Moody Syrnmetry of the Geometric Actions

Aratyn, E. Nissimov and S. Pacheva

UICHEP-TH/90-15

July 1990, 10pp, to be published in Mod. Phys. Lett. A

A12. Coset Construction of Superstrings via the Coadjoint Orbit Method Aratyn, E. Nissimov and S. Pacheva

UICHEP-TH/90-16

Aug. 1990, 9pp, to be published in Mod. Phys. Lett. A

A13. Kac-Moody Construction of Toda Type Field Theories

Aratyn, L.A. Ferrela, J.F. Gomes and A.H. Zimerman

UICHEP-TH $/ 90-17$

Sept. 1990, 11pp. to be published in Phys. Lett. B

A14. Infinite-Dimensional Noether Symmetry Groups and Quantum Effective Actions from Geometry

Aratyn, E. Nissimov and S. Pacheva

UICHEP.TH/90-20

Sept. $1990,11 \mathrm{pp}$ submitted for publication

B1. Statistics of 2D Solitons and 3D Vortices

Brekke and $T$. Imbo

Univ. of Illinois at Chicago preprint UICHEP-TH/90-21 (:\$30)

B2. Anyonic Nielsen-Olesen Vorrices

Brekke, A.F. Falk, S.J. Hughes and T. Imbo

Univ. of Illinois at Chicago preprint UICHEP-TH/90-22 (1990)

K1. Higgs-Boson-Mediated Neutrinoless Double Beta-Decay and Neutrino Mass in a Majoron Model

Keung and $D$. Chang

Phys. Rev.. D39, 1386-1390 (1989)

K2. Constraints on Muonium-Antimuonium Conversion

Keung and $D$. Chang

Phys. Rev. Lett. 62, 2583-2585 (1989)

K3. Z Boson Bremsstrahluny in Heavy Quark Decay

Keung, $V$. Barger and T. Rizzo

Phys. Rev. D40 2274-2278 (1989) 
K4. Flavor--Changing Decays of Fourth Generation Quarkonium Keung and $V$. Barger

Phys. Rev. 40, 2941-2943 (1989)

K5. Z-Boson Decays to Heavy Quarkonlum Keung, V. Barger and K. Cheung

Phys. Rev. D41, 1541 (1990)

K6. QCD Corrections to CP Violation from the Color Electric Dipole Mornent of the b Quark

Keung, D. Chang, C.S. LI, and T.C. Yuan

Phys. Lett. 241B, 589-592 (1990)

K7. Gauge Hlerachy and Attractive Feeble Long-Range Force

Keung, $D$. Chang and $P$. Palash

Phys. Rev. D42, 630-635 (1990)

K8. Neutrino Magnetic Moment and Nonabelian Discrete Symmetry

Keung, D. Chang and $G$. Senjanovic

Phys. Rev. D42, 1599-1603 (1990)

K9. Photon Bremsstrahlung In Heavy-Top-Quark Decay

Keung, $V$. Barger and $A$. Strange

Phys. Rev. D42, 1835-1936 (1990)

K10. Two Loop Bosonic Contribution to the Electron Electric Dipole Moment

Keung, D. Chang and T.C. Yuan

submitted to Phys. Rev. D (1990)

K11. The Electric Dipole Moment of W Boson

Keung, D. Chang, J. Liu

submitted to Nucl. Phys. B (1990)

K12. Chromoelectric Dipole Moment of Light Quarks Through Two Loop Mechanism Keung, D. Chang and T.C. Yuan

submitted to Phys. Lett. B (1990)

P1. Radiative Symmetry Breaking in $(2+1)$-dimensional Space

Panigrahi, K. S. Babu and S. Ramaswamy

Phys. Rev. D39, 1190 (1989)

Pź. Canonical Structure of the O(3)a Non-linear Sigma Model with a Topological Term Panigrahi, T. L. Olczyk and S. Ramaswamy

Z. Phys. C45, 653-656 (1990)

P3. Radiative Decay of Mesons and Anomaly in $2+1$ Dimensions

Panigrahi, T. L. Olczyk and 0 . Roy

to appear in Z. Phys. C (1990) 
P4. Effective Lagrangian for a System of Non-relativistic Fermions in $2+1$ Dimensions Coupled to an Electromagnetic Fleld: Application to Anyonic Superconductors

Panigrahi, R. Ray and B. Sakita

submitted to Phys. Rev. B42, 4036 (1990)

P5. Covariant Operators, Higher Spin Conformal Algebras and the Boussinesq Sequence

Panigrahi, Q. Wang, U. Sukhatme and W.-Y. Keung

Nucl. Phys. B344, 196 (1990)

S1. Phase Equivalent Potentials Obtained from Supersymmetry

Sukhatme and $A$. Khare

Jour. Phys. A22, 2847 (1989)

S2. Families of Strictly Isospectral Potentials

Sukhatme, W.-Y. Keung, Q. M. Wang and T. Imbo

Jour. Phys. A22, L987 (1989)

S3. Countable Infinity of Isospectral Potential Families

Sukhatme and $A$. Khare

Phys. Rev. A40, 6185 (1989)

S4. Solitons from Supersymmetry

Sukhatme, Q. M. Wang, W.-Y. Keung and T. Imbo

Mod. Phys. Lett. A5, 525 (1990)

S5. Supersymmetry Inspired WKB Approximation in Quantum Mechanics

Sukhatme, R. Dutt and A. Khare

Univ: of Illinois at Chicago preprint UICPHY-TH/90-4 (1990)

S6. Analog of the EMC Effect in Neutrino-Nucleus Interactions

Sukhatme and $K$. Lassila

Int. Jour. Mod. Phys. A (1990)

S7: Small--x Shadowing in Dimuon Production

(with K. Lassila)

Univ. of Illinois at Chicago preprint UICHEP-TH/90-8 (1990)

S8. Dilepton Production by Protons on Nuclei and the Partonic Origin of Depletion at. Small Momentum Fraction

(with K. Lassila, A. Harindranath and J. Vary)

Univ. of Illinois at Chicago preprint UICHEP-TH/90-11 (1990)

s9. Gluon Structure Functions in Nuclel from the Quark Cluster Model (with G. Wilk and K. Lassila)

Univ. of Illinois at Chicago preprint UICHEP-TH/90-10 (1990)

S10. Backward Hadrons from Deep Inelastic Lepton Scattering on Nuclei (with C.E. Carlson and K. Lassila)

Univ. of Illinois at Chicago preprint UICHEP-TH/90-18 (1990) 
S11. Nuclear Effects in the Hadroproduction of Charm Sukhatme, P. Hoyer, B. P. Mahapatra and K. Sridhar Phenomenology of the Standard Model and Beyond Proc. Workshop on High Energy Physics Phenomenology, TIFR, Bombay (1989), edited by D. P. Roy and P. Roy, p. 173

S12. Violation of Factorization in Charm Hadroproduction (with P. Hoyer and M. Vanttinen)

Phys. Lett. 246B, 217 (1990)

S13. Finite Eigenfunctions in the WKB Approximation (with A. Pagnamenta)

Univ. of Illinois at Chicago preprint UICHEP-TH/90-5 (1990)

S14. Non-Divergent Semiclassical Wave Functions in Supersymmetric Quantum Mechanics (with A: Pagnamenta)

Univ. of Illinois at Chicago preprint UICHEP-TH/90-9 (1990);

to appear in Phys. Lett. A 
- We give below a brief description of the main areas in which new physics results were obtained.

I. H. Aratyn

A. BRS Operator and Physical States of the String Model (A1,A3-A6)

We found the abelian representation of the string BRS cohomology in terms of basic operators forming naturally two dual Kugo-Oljima quartets. Furthermore, using our gauge-fixing scheme, we constructed a method providing the explicit realizations of both the physical and unphysical subspaces for the string theory.

The passage between abelian and nonabelian quantities was realized geometrically through the use of the moments of the vertex operator, which acted as vielbeins between infinite-dimensional algebras. We have clarifled the connection between the Virasoro and the spectrum-generating algebra and uncovered the algebraic duality relation between them.

\section{B. Covariant BRS QLiantization of the Green-Schwarz Superstring (A2)}

The superstring action can be written in two different ways. The corresponding actions are: called, respectively, the Neveu-Schwarz-Ramond and the Green-Schwarz actions. The Neveu-Schwarz-Ramond formalism allows covariant quantization but the space-time supersymmetry is obscure in this model. On the other hand, the GreenSchwarz action possesses the attractive feature of being manifestly supersymmetric. For this reason there has recently been an extensive amount of work dedicated to the Investigation of quantizing the Green-Schwarz superstring in the covariant manner and keeping the supersymmetry manifest. Although the light-cone quantization of this model was easily performed the covarlant quantization has presented formidable difficulties, due to the presence of both first and second class constraints and the difficulty in separating them covariantly. This lack of covarlant quantization of the superstring model is highly unsatisfactory and called therefore for invention of new quantization scheme for dealing with second class constraints. As a step in this direction we have performed the covariant BRS quantization of the $N=1$ superparticle prototype of the Gieen-Schwarz superstring in ten dimensions. One of the key ideas was addition of the auxiliary variables according to the Batalin-Fradkin formallsm for the second class constraints.

C. Construction of the Invariant Actions by the Method of Coadjoint Orbits (A7-

Recently we have extended the method of coadjoint orbits of infinite-dimensional Lie groups for derivation of geometric $D=2$ field theory actions to the super-KacMoody and super-Virasoro groups in a manifestly supersymmetric form. In this way we 
derive the explicit Expressions for the actions of the supersymmetric chiral Wess-ZuminoNovikov-Witten (WZNW) model and of the induced supergravity (the super-gravitational Polyakov action).

We have also derived a new formula to produce yeneral actions in this framework only in terms of the basic group theoretical objects. The method was lllustrated in the number of examples including extended superconformal models.

D. Noether Theorem for Geometric Actions and the Area Preserving Diffeomorphlsms on Torus (A9)

We found that within the formalism of coadjoint orbits of the infinite dimensional Lle group the Noether procedure leads, for a special class of transformations, to the constant of motion given by ihe fundamental group one-cocyle $\mathbf{S}$. Use is made of the simplified formula giving the symplectic action in terms of $\mathbf{S}$ and the Maurer-Cartan one-form.

The area preserving diffeomorphisms on the torus $T^{2}=S^{1}(x) S^{1}$ constitute an algebra with central extension, given by the Floratos-Illopoulos cocycle. We applied our general treatment based on the symplectic analysis of coadjoint orbits of Lie groups to write the symplectic action for this model and study its invariance. We found an interesting abelian symmetry structure of this non-linear problem.

E. On the Group Theoretical Meaning of the Conformal Field Theories in the Framework of Coadjoint Orbits (A10)

We presented an unifying approach to conformal fleld theories and other geometric models within the formalism of coadjoint orbits of infinite dimensional Lle groups with central extensions. Starting from the previously obtained general formula for the symplectic action in terms of two fundamental group one-cocyles, we derived the most general form of the Polyakov-Wlegmann composition laws for any geometric model. These composition laws are succinct expressions for all pertinent Noether symmetries As a basic consequence we obtain Ward identities allowing for making further progress in determining the quantum propertles of the geometric models.

\section{F. Hidden Kac-Moody of the Geometric Models (A11)}

A general formalism was proposed to study Infinite-dimensional Noether symmetries In arbitrary field theories on group coadjoint orbits as well as in their gauged versions (coset geometric models). The basic tools are generalized group composition laws valid for any geometric action. As a main application, we presented a general scheme for constructing the "hidden" Kac-Moody currents. 
G. Coset Construction of Superstrings via the Coadjoint Orbit Niethod (A12)

The previously proposed general construction of geometric actions on infinitedimensional group coadjoint orblts in terms of fundamental group one-cocycles was applied to provide an alternative formulation of the Green-Schwarz superstring. It was shown that the latter model can be consistently constructed as geometric action on a certain infinite-dimersional coset space of the semidirect product of left- and righthanded Virasoro groups with a Kac-Moody group based on an appropriate modification of the super-Heisenberg-Weyl group.

H. Kac-Moody Construction of Toda Type Fleld Theorles (A13)

Using the coadjuint orbit method we derived a geometric WZWN action based on the extended two-loop Kac-Moody algebra, which we had introduced for the purpose of study models exhibiting both conformal invarlance and integrabllity. We have shown that under a Hamiltonian reduction procedure, which respects conformal invariance, one obtains a hierarchy to Toda type fleld theorles, containing as submodels the Toda molecule and perlodic Toda lattice theories. We also discussed the classical $r$-matrix and the integrabllity properties of resulting models.

I. Infinite-Dimensional Noether Symmetry Groups and Quantum Effective Actions from Geometry (A14)

We have considered quantum effective actions for arbitrary models possessing an infinite-dimensional group $G$ of Noether symmetries. We observed that the relevant Ward identities yleld functional differential equations for the effective action whose exact solution is found to be given by the geometric action on a coadjoint orblt of the (central extended) Noether group G. As a particular application we show that the effective action of the light-cone quantized toroidal membrane is explicitly given by the geometric co-orbit action of the group of area-preserving diffeomorphisms on torus.

Starting from the general symplectic manifold's formallsm (generalizing the group coadjoint orbit formalism) we discussed arbitrary models (in $D \geqslant 2$ dimensions) possessing an Infinite-dimensional group $G$ of Noether symmetries and consider coupling of the Noether currents to external "sources". We wrote down the Ward Identitles for the corresponding quantum effective actions and employed the $W-Z$ consistency relations to uncover the following remarkable property: no matter what is the specific action of the initial classical model, its quantum effective action is always given by the geometric action on a generic coadjoint orbit of the (central extension of the) Noether symmetry group $\widetilde{G}$. Given therefore the model with Noether symmetry algebra which admits central extension, the geometric action approach developed by us can then be employed to find its unique quantum effective action. 
'II. L. Brekke

A. Various Aspects of Statistics of Solitons (B1-B2)

In the preprint $B 1$, "Statistics of $2 \mathrm{D}$ solitons and $3 \mathrm{D}$ vortices", we give a general technique for defining and calculating the possible statistics of solitons in $1+1$ dimensional sigma models (with space a circle) and of vortices in spontaneously broken gauge theories in $2+1$ dimensions. These results also apply to cosmic strings in $3+1$ dimensional gauge theories. Several specific examples were given - some of which give solltons with an exotic form of statistics known as ambistatistics. This form of statistics effectively violates the superselection rule between Bose and Fermi statistics. One model which admits ambistatistical vortices is the "Alice vortex" model prevlously studied by Coleman and collaborators. Models with fractional ambistatistics were also found.

In the preprint B2, "Anyonic Nielsen-Olesen Vortices", we explicitly display a topological term which gives fractional statistics to the Nielsen-Olesen vortex of the abellan Higgs model in $2+1$ dimensions. The resulting anyons are different from anyons created by adding a Chern-Simons term to the action in that they are not charged. The statistics of composites of anyons are also different in the two cases.

III. W.-Y. Keung

A. Symmetries in Gauge Theories (K1,K2,K7,K8)

(i) We have studied a simple model in which the muonlum-antimuonium conversion can occur at a level around the recent experimental bound but the process $\mu \rightarrow$ er is strictly forbidden. Measurements of the anomalous muon magnetic moment and the high energy Bhabha scattering $\theta^{+} \theta^{-} \rightarrow \theta^{+} \theta^{-}$together provide an indirect and interesting constraint on the conversion. The model predicts anomalous events ep $\rightarrow \overline{\theta \mu} \mu \mathrm{X}$ at $\mathrm{hi}$ h $h$ energy ep colliders. (ii) We have related the long range of an attractive feeble force to the gauge hierachy in grand unification. (iii) We have studied a mechanism which gives a small mass but a large magnetic moment for the neutrino such that the solar neutrino deficit problem can be explinineci. The idea is a discrete version of Voloshin's SU(2) mechanism. An example using the quaternion group is illustrated.

B. Collider Physics (K3-K5,K9)

(1) The decay of a massive top quark accompanied by the emission of a "hard" photon or a $Z$ boson has been studied. This process may be used to tost the trillinear gauge boson coupling of the standaru model. (ii) The decay of a fourth generation quarkonium Into flavor-changing channels cte etc. has been shown to occur at a branching fraction of order $1 \%$ under favorable mass and mixing cinditions, (iii) We also studled the inclusive quarkonium production in $Z$ decays. 


\section{Electric Dipole Moments (K6,K10-K12)}

Following the recent works of Welnberg, and Barr and Zee on the electric dlpole momerts of the neutron and the electron, we have set up the correct QCD evolution equation for the relevant operators, in particular, the chromoelectrlc dipole moments of quarks which play an important role in the neutron electric dipole moment. For the electron dipole moment, we have performed a general analysis in multi (>2)-Higgs models, with technical calculations of the two-loop contribution.

\section{P. Panigrahi}

A. Radiative Symmetry Breaking in $(2+1)$ Dimensional Space (P1)

A systematic study of the one-loop effective potential in $U(1)$ invariant theories involving scalars, fermions and gauge flelds in $(2+1)$ dimensional space-time was carried out. Radiative symmetry breaking was investigated. Both for ordinary and Chern-Simons QED, no radlative symmetry breaking occurs, if this theory is scale invariant at the tree level. However the symmetry always breaks down, when the scalars are coupled to charged fermions. At a sufficiently high temperature symmetry is again restored. B. Canonical Structure of the $O(3)$ Non-linear Sigma Model with a Topological
Term (P2)

In $1+1$ space-time the $O(3)$ non-linear $\sigma$-model with a topological term was quantized following Dirac's method of quantization. The current algebra was investigated for a specific value of this coefficient of the topological term. We showed the equivalence of this algebra with $k \rightarrow \infty$ limit of the algebra of $S U(2) \times S U(2)$ Invariant WZW model. The operator ordering ambiguities were resolved following the prescriptions of nonlinear quantum mechanics and the quantum Hamiltonian was obtained.

\section{Radiative Decay of Mesons and Anomaly in 2+1 Dimensions (P3)}

Decay of mesons coupled to fermions by Yukawa couplings was studied in two dimensional space. In a four component theory, the electromagnetic current is free of parity odd radiative corrections; however an axial $U(1)$ current is shown to develop a finite vacuum expectation value. Unlike even dimensions this anomaly is temperature dependent and does not play any role in the radiative decay. We calculate the effective action for the mesons and show that a finite kinetic term for these flelds is induced at the one-loop level and vanishes as the temperature goes to infinity. 
D. Effective Lagrangian for a System of Non-relativistic Fermions in the Presence of Electromagnetic Field: Application to Anyonic Superconductors (P4)

The effective astion for non-relativistic fermions was computed in the presence of background electromagnetic field. The one loop action yields a Chern-Simons like term. The results were then applied to the system of anyons. We showed that the canceliation of the inducec, C-S term against a C-S term included from the beginning to change the fermions to anyons is favoured energetically, together with the expulsion of $B(x)$ from the system of anyons. A massless collective mode, representing the density fluctuation is shown to exist.

E. Covariant Operators, Higher Spin Conformal Algebras and the Boussinesq Sequence (P5)

Operators transforming covariantly under the action of Diff $S^{1}$ are used as Lax operators to study the second Hamiltonian structure of the Lax equations. The classical spin-4 algebra is explicitly obtained. The associated non-linear equations are also studied. The Boussinesq sequence arised naturally for the spin-3 case.

\section{U. Sukhatme}

\section{A. Multi-Soliton Solutions and Supersymmetric Quantum Mechanics (S1-S5)}

There have been a large number of publications in the past few years dealing with the application of supersymmetry to quantum mechanics. This has ylelded a deeper understanding of analytically solvable potentials as well as improvements of approximation techniques like large $N$ expansions and the WKB method. In particular, supersymmetric quantum mechanics provides a powerful new way of constructing families of isospectral potentials. Given any potential, we have solved the problem of finding the most general family of potentials which has the same bound state energies and scattering amplitudes. It turns out that this family has $n$ parameters, where $n$ is the number of bound states in the potential. Such families are known to solve nonlinear differential equations of physical interest e.g. Korteweg-de Vries equation. By suitably identifying the $n$ parameters in our isospectral families, we have obtained explicit, simple expressions for pure multi-soliton solutions [corresponding to reflectionless potentials].

B. Effects of Quark Clusters in Nuclei (S6-S10)

One of the successful phenomenological explanations of the EMC effect ( $\mathrm{C}^{-}$- deep inelastic lepton scattering on nuclei) is based on the formation of "quark clusters" in nuclei. It is natural to look at other processes involving ruclei for related phenomena. We have investigated the consequences of clusters in neutrino and antineutrino charged current events. Although, in principle, discriminating neutrino experiments which empha- 
'size parton structure functions in different momentum fraction regions are possible, unfortunately accurate data will be hard to obtain. The situation is quite different for the Drell-Yan process. The shadowing behavior at small-x predicted by the quark cluster model has recently been confirmed by dimuon production measurements for protons incident on nuclel [experiment E772 at Fermilab]. The model also predicts that gluon and ocean quark structure functions in nuclel are shadowed but the valence quarks are not. We have also calculated the spectrum of hadrons produced in the backward hemisphere from deep inelastic lepton scattering on nuclear targets.

C. Nuclear Effects in the Hadroproduction of Charm (S11-S12)

Several experiments on open charm and $J / \psi$ hadroproduction suggest that the steepness of the Feynman $X_{F}$-distribution of charm increases markedly with the size of the nuclear target. Furthermore, this A-dependence of the $x_{F}$-distribution is observed to be essentially indeperident of energy. We have shown that the existing data are incompatible with the standard lowest order $Q C D$ factorization formula, which predicts the $x_{F}$-distribution to be essentially determined by the gluon structure function of the beam. This implies that charm hadroproduction contains substantial contributions from "higher twist" effects.

D. Non-Divergent Semiclassical Wavefunctions (S13-S14)

A long-standirig problem and major shortcoming of semiclassical (WKB) bound state wave functions is their divergence at the classical turning points. Presently available regularization schemes are accurate but rather complicated. We show how finite wave functions can be simply obtained by reorganizing the WKB perturbation expansion in powers of $h$ and retaining appropriate higher order terms. This technique has been applied both to the ordinary WKB as well as the supersymmetric WKB approximation. The resulting finite wave functions compare well with numerical results, and provide a useful starting point for the calculation of physically interesting matrix elements. 

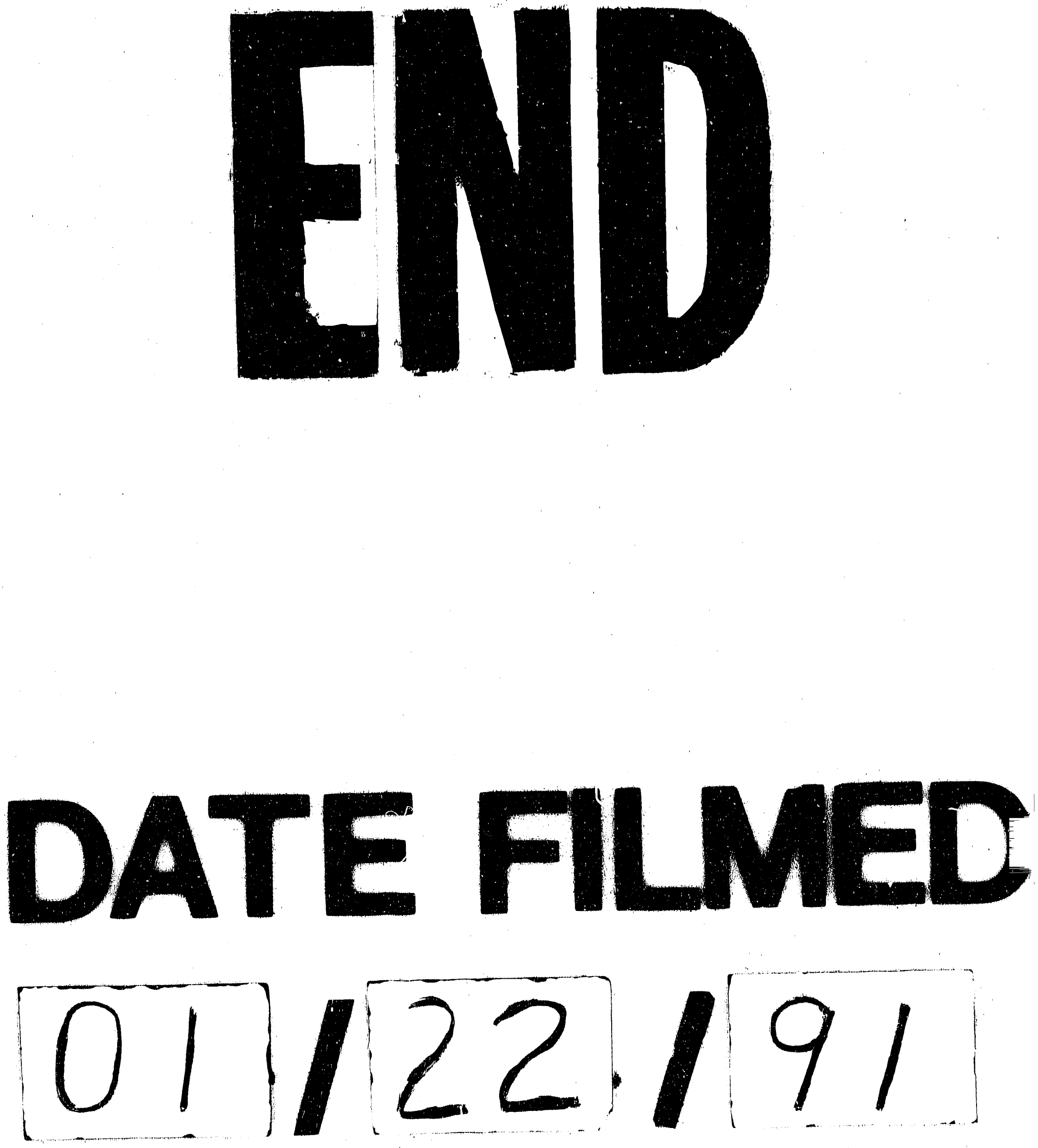
